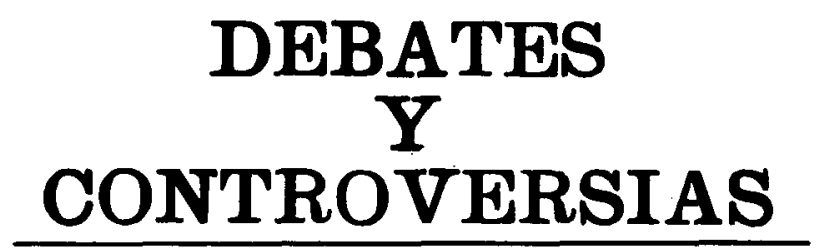




\section{LA ELECTRICIDAD EN ESPAÑA ANTES DE LA GUERRA CIVIL: UNA REPLICA}

CARLES SUDRIA

Universidad de Barcelona

\section{Introducción}

En un reciente artículo en esta misma Revista sobre la introducción de la electricidad en España durante el primer tercio del presente siglo, Francesca Antolín ha presentado una nueva aproximación, incluyendo nueva evidencia empírica y nuevos elementos de análisis '. Algunas de las hipótesis que se sostienen en ese trabajo son, sin duda, atrayentes y susceptibles de inspirar nuevas investigaciones, muy en especial en el ámbito de la estrategia empresarial. Otras propuestas, sin embargo, contradicen, a mi entender sin base suficiente, explicaciones propuestas hace algún tiempo por Jordi Maluquer de Motes, y que yo mismo he recogido en diversos trabajos ${ }^{2}$.

Maluquer y yo hemos venido sosteniendo que la posibilidad de transportar el fluido eléctrico a largas distancias permirió, en España como en otroś países, la utilización a gran escala de la energía hidráulica para la producción de electricidad. Mediante una renovación total de la propia estructura del sector eléctrico y después de un proceso relativamente rápido, la electrificación se difundió por la mayor parte del aparato productivo español, alcanzándose, en los años previos a la Guerra Civil, cotas com. parables a las de otros países más avanzados. Estas conclusiones se apoyaban en una serie de informaciones cualitativas y cuantitativas - a las que luego me referiré- $y$ en un razonamiento que parecía defendible: si la economía española había tenido que soportar durante el siglo XIX unos costes comparativamente elevados de la energía que derivaba del carbón mineral, resultaba lógico que se adhiriera con rapidez e intensidad a una innovación que la liberaba parcialmente de aquella costosa dependencia.

Estas conclusiones son rebatidas por Antolín. En sus propias palabras, en España, «a un distanciamiento muy modesto entre precios relativos [de la

'Antolín (1988).

2 Maluquer de Motes (1983, 1985 y 1987) y Sudrià (1987a, 1987 b y $1987 c)$. 
electricidad y el carbón mineral] le corresponde un proceso de avance de la electrificación muy lento respecto al vapor y poco radical en cuanto a la estructura interna del sector productor» ${ }^{3}$. Para demostrarlo se realiza una comparación sistemática del caso español — con especial referencia al País Vascocon el caso norteamericano.

Las razones de la discrepancia entre las dos visiones del tema radican en la adopción de términos de comparación y de métodos de câlculo diferentes. Es conveniente por ello discutir uno por uno los puntos de desacuerdo, aportando todos los datos disponibles.

\section{Los precios absolutos y los precios relativos}

El punto de partida de la argumentación de Antolín es la afirmación de que los precios de la electricidad y los del carbón mineral evolucionaron en España de forma mucho menos discrepante que en Estados Unidos. En concreto, se establece que entre 1913 y 1929, en España, los precios del carbón se multiplicaron por 1,7 respecto a los de la electricidad, mientras en los Estados Unidos el mismo multiplicador se situó en 6 . Frente a esta afirmación aparecen dos tipos de cuestiones. La primera afecta a las fuentes utilizadas, y se discutirá más abajo. La segunda es de carácter lógico. El estudio de la evolución de los precios relativos en base a un índice puede resultar completamente engañoso si en el punto de partida, 1913 en este caso, la relación entre los precios del carbón y de la electricidad en ambos países fuera muy distinta. En otras palabras, puede ser que el uso de una de las fuentes energéticas fuera desde el inicio más ventajoso en un lugar que en otro. El cálculo debe realizarse utilizando los precios reales, no los números índice.

El problema de los precios de la electricidad es extremadamente complejo. La razón de ello es que no existía un precio único, sino varios, a menudo muy diferentes, en función del tipo de consumo y de la potencia contratada. En general, la información disponible se refiere a tarifas domésticas y no a las de consumo industrial, sin duda muy inferiores. Las cifras españolas utilizadas por Antolín proceden del Anuario Estadístico, y las norteamericanas las toma del artículo de A. G. Woolf en el que inspira su trabajo ${ }^{4}$. Woolf, sin embargo, ofrece tan sólo una aproximación, remitiendo a diversas fuentes primarias que, al parecer, no han sido consultadas por Antolín. Una de estas fuentes son las Historical Statistics of the United Siates, según las cuales el citado multiplicador del precio relativo se situaría en 2,1 , muy cercano al es-

\footnotetext{
3 Antolín (1988), p. 648.

- Woolf (1984), p. 178.
} 
pañol ${ }^{5}$. Cálculos similares para Francia e Italia ofrecen una variación de los precios relativos del mismo orden moderado (cuadro 1).

\section{CUADRO 1}

Evolución de los precios de la bulla y de la electricidad en diversos países

\begin{tabular}{|c|c|c|c|c|c|c|}
\hline \multirow{5}{*}{$\begin{array}{l}1913 . \\
1929 . \\
1929 / 1913\end{array}$} & \multicolumn{3}{|c|}{ EE. UU. } & \multicolumn{3}{|c|}{ FRANCIA } \\
\hline & $\begin{array}{c}\text { Elect. } \\
(\$ / \text { kwh })\end{array}$ & $\begin{array}{l}\text { Hulla } \\
(\$ / t)\end{array}$ & $\begin{array}{l}\text { Precio rel. } \\
\text { bulla/elect. }\end{array}$ & $\begin{array}{c}\text { Elect. } \\
(F / \text { kwb })\end{array}$ & $\begin{array}{l}\text { Hulla } \\
(F / t)\end{array}$ & $\begin{array}{l}\text { Precio rel. } \\
\text { bulla/elect. }\end{array}$ \\
\hline & $\begin{array}{l}0,09(1) \\
0,06\end{array}$ & $\begin{array}{l}1,18 \\
1,78\end{array}$ & $\begin{array}{r}13,0 \\
28,2 \\
2,1\end{array}$ & $\begin{array}{l}0,57(2) \\
1,60\end{array}$ & $\begin{array}{r}24,5 \\
136,0\end{array}$ & $\begin{array}{r}43,0 \\
85,0 \\
2,0\end{array}$ \\
\hline & \multicolumn{3}{|c|}{ ITALIA } & \multicolumn{3}{|c|}{ ESPAÑA } \\
\hline & $\begin{array}{l}\text { Elect. } \\
(L / k w b)\end{array}$ & $\begin{array}{l}\text { Hulla } \\
(L / t)\end{array}$ & $\begin{array}{l}\text { Precio rel. } \\
\text { bulla/elect. }\end{array}$ & $\begin{array}{c}\text { Elect. } \\
(\text { ptas } / k w b)\end{array}$ & $\begin{array}{c}\text { Hulla } \\
(\text { ptas } / t)\end{array}$ & $\begin{array}{l}\text { Precio rel. } \\
\text { bulla/elect. }\end{array}$ \\
\hline $\begin{array}{l}1913 . \\
1929 \text {. } \\
1929 / 1913\end{array}$ & $\begin{array}{l}0,53(3) \\
1,60\end{array}$ & $\begin{array}{r}30(4) \\
150(5)\end{array}$ & $\begin{array}{r}56,6 \\
93,8 \\
1,7\end{array}$ & $\begin{array}{l}0,21 \\
0,22\end{array}$ & $\begin{array}{l}20 \\
35\end{array}$ & $\begin{array}{r}95,2 \\
159,1 \\
1,7\end{array}$ \\
\hline (1) 1912 . & (2) 1914. & (3) 1911 & (4) & 1901-10. & $1921-3$ & \\
\hline
\end{tabular}

Pese a las precauciones con que hay que utilizar estas cifras, todo parece avalar dos hipótesis: a) la evolución de los precios relativos en los diversos países fue más o menos similar, y $b$ ) desde el mismo inicio de la electrificación, en España, Italia y Francia la ventaja comparativa de la electricidad era mucho más amplia que en los EE. UU. Esto se corresponde perfectamente con el hecho bien conocido de que los Países mediterráneos están mejor dotados, en términos relativos, de energía hidráulica que de recursos carboníferos. Parece claro, pues, que españoles, italianos y franceses con-

${ }^{5} \mathrm{Mi}$ cálculo corresponde a precios medios nacionales. Pese a que no lo indica, es posible que el trabajo de Woolf se refiera a una zona determinada o a un tipo específico de consumidores. En todo caso, serían las cifras medias las comparables con las españolas. 
taron desde el primer momento con mayores estímulos para sustituir las energías derivadas del carbón por la electricidad.

\section{3. ¿Se electrificó realmente España?}

La segunda de las conclusiones formuladas por Antolín se refiere al grado de electrificación alcanzado por España antes de la Guerra Civil. Según ella, el avance de la electricidad respecto a la energía tradicional del vapor fue en España débil en comparación con el registrado en los Estados Unidos. Para sostener esta afirmación, Antolín ofrece datos sobre la potencia instalada en las grandes empresas metalúrgicas de Vizcaya y Guipúzcoa, deduciendo de ellas que hacia 1930 sólo un 30 por 100 de la potencia instalada correspondía a motores eléctricos. Esto se contrasta con las cifras estadounidenses correspondientes a 1929 , calculadas por R. B. DuBoff y recogidas por Devine, que muestran una aportación de los motores eléctricos del 78 por 100 de toda la potencia instalada en la industria manufacturera ${ }^{6}$.

El problema reside, en este caso, en no ser las cifras que se presentan directamente comparables. En primer lugar, y para ajustar la potencia nominal de los motores a la realmente activa, Antolín ha dividido por cinco la corres. pondiente a los motores eléctricos. Tal reducción, sin embargo, no se ha aplicado a las cifras estadounidenses con las que establece la comparación. En segundo lugar, es sabido que en el ámbito del sector del metal - tanto en Estados Unidos como en España - una parte muy importante de la energía eléctrica utilizada se produce en las propias fábricas metalúrgicas, aprovechando el calor y los gases generados en los procesos de producción. Existen así dos tipos de motores, aquellos que se utilizan directamente para accionar la maquinaria y aquellos que se utilizan para generar electricidad. Si se suman unos y otros motores, como hace Antolín para el caso de las provincias de Vizcaya y Guipúzcoa pero no las fuentes estadounidenses, se produce una duplicación parcial de la potencia instalada. Como las fuentes españolas no permiten realizar la distinción, hemos adaptado las norteamericanas al mismo sistema de cálculo. Recurriendo de nuevo a las fuentes originales y teniendo en cuenta lo antedicho (cuadro 2) vemos que, hacia 1930, prácticamente no existía diferencia real entre el grado de electrificación de la industria metalúrgica española y la de los EE. UU.

' Devine (1983), p. 351. 


\section{CUADRO 2 \\ Potencia de la maquinaria instalada en el sector metalúrgico (Miles de CV)}

\begin{tabular}{|c|c|c|c|}
\hline & $\begin{array}{c}\text { Total incluyendo } \\
\text { la autoproducción } \\
\text { eléctrica }\end{array}$ & $\begin{array}{l}\text { Motores } \\
\text { eléctricos }\end{array}$ & $\begin{array}{l}\text { Porcentaje } \\
\text { de motores eléctricos } \\
\text { sobre el total }\end{array}$ \\
\hline Vizcaya $(1930)$ & 151,8 & 105,3 & 69,3 \\
\hline Guipúzcoa (1930) ... & 16,5 & 9,1 & 55,4 \\
\hline EE. UU. (1929) & 18.789 & 12.251 & 65,3 \\
\hline
\end{tabular}

Fuentes: Antolín (1988), p. 647, y Bureau of Census (1933), Abstract of the Fifteenth Census of the United States, pp. 824-827. Las cifras correspondientes a toda la industria manufacturera ofrecidas por esta fuente presentan muy leves diferencias con las publicadas por Devine (1983), salvo por el hecho de excluir éste los motores térmicos destinados a producir electricidad.

Al margen de estos problemas, es obvio que una comparación de este tipo está directamente afectada por la estructura interna del sector en cada país y, en todo caso, no es generalizable al conjunto de las actividades económicas. Una forma mucho más fiable de aproximarse a la importancia relativa del con. sumo eléctrico es relacionándolo con el consumo energético total. En concreto, podríamos confirmar la hipótesis formulada por Antolín, según la cual la expansión de la electricidad respecto al vapor fue más lenta en España que en otros países, si en los años 1930 observaríamos una menor importancia de la electricidad dentro del consumo energético total. El cuadro 3 -fila $b$ ) muestra que el caso era exactamente el inverso. En 1929, el 17,5 por 100 del consumo energético español se realizaba a través de la electricidad. Un porcentaje sólo superado por Italia y un 50 por 100 superior al norteamericano. Resulta claro que, en la competencia con el vapor, la electrificación española había avanzado más que la norteamericana. Nada más lógico si tenemos en cuenta los menores costos relativos que presentaba la electricidad tanto en España como en Italia.

Que la electricidad estuviera ocupando en España un espacio más amplio dentro del consumo energético no significa, claro es, que se consumiera más electricidad en España que en otros países. El mismo cuadro 3 recoge una serie de comparaciones cuyo comentario puede resultar útil. Se observa, en primer lugar, que España mantenía un nivel de consumo de energía por unidad de producto relativamente bajo - fila $f$ ) - Esto corresponde al mayor 


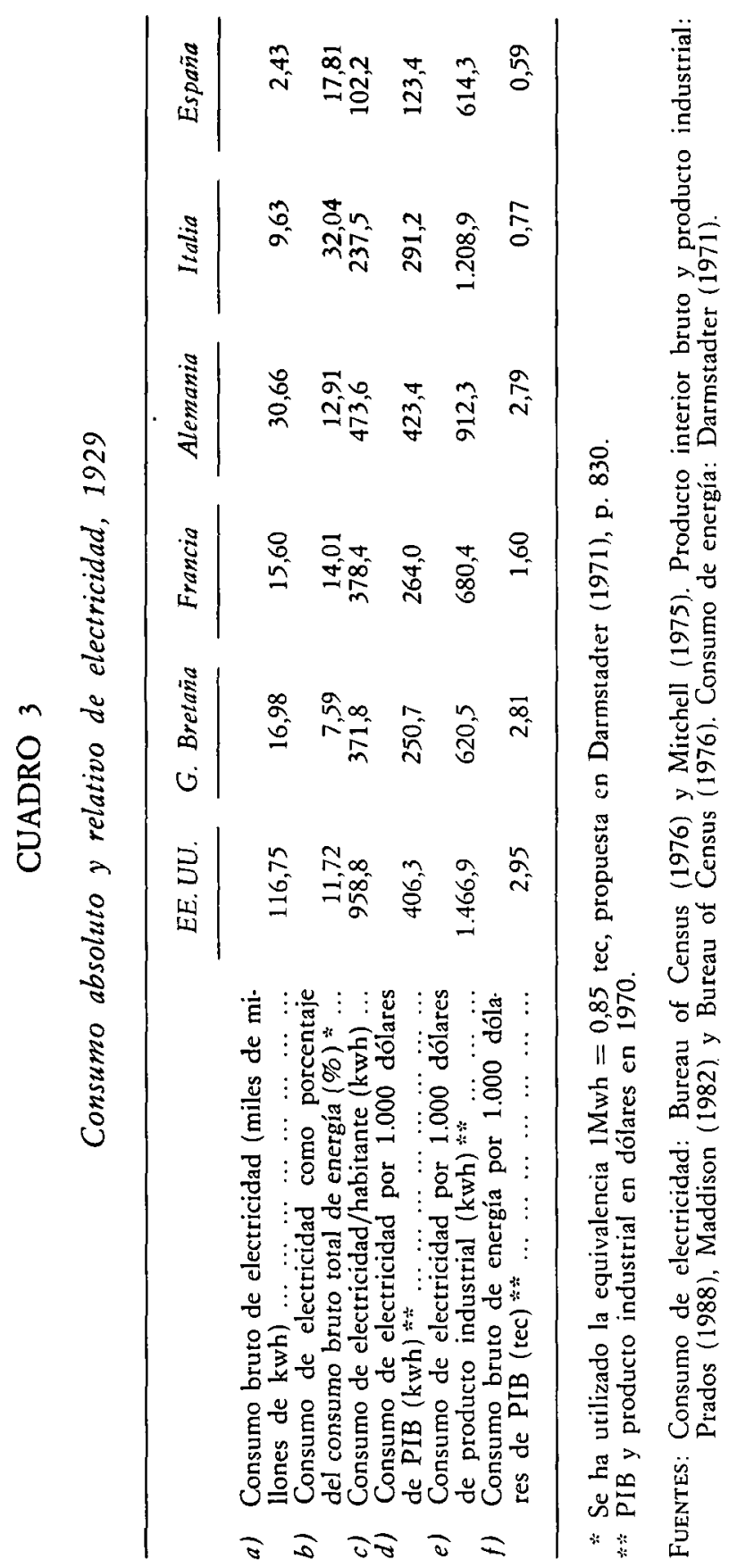


peso que dentro del PIB español tenían la agricultura y otras actividades de bajo consumo energético. Dentro mismo del sector industrial, el coste elevado de la energía había perjudicado el desarrollo de las producciones con mayores requerimientos energéticos ${ }^{7}$.

Si atendemos específicamente a la electricidad veremos que el consumo español por habitante y por unidad de producto - filas $c$ ) y $d$ ) - era notoriamente inferior al de los otros países considerados. Sin embargo, si excluimos del cálculo el sesgo introducido por la menor industrialización de España, relacionando el consumo de electricidad con el producto industrial - fila $e$ ) las diferencias se reducen notablemente ${ }^{8}$. En concreto, España queda situada al mismo nivel de Gran Bretaña y Francia, aunque alejada de Estados Unidos e Italia. Observemos que lo que muestra este indicador es la importancia de la electricidad respecto a la producción industrial, pero no de la electricidad respecto a otras fuentes de energía. En definitiva, la industria de algunos de los países de mayor desarrollo, como los EE. UU. y Alemania, consumía más energía total y más energía eléctrica por unidad de producto que la española, pero el peso de la electricidad dentro de su consumo energético total era menor que en España.

\section{La producción de electricidad en España: ¿un sector atomizado?}

La última de las afirmaciones de Francesca Antolín que quiero discutir hace referencia a la estructura del sector productor de electricidad. Según sus palabras, citadas más arriba, el avance observado entre principios de siglo y 1930 sería «poco radical en cuanto a la estructura interna del sector productor». El bajo nivel de concentración alcanzado conllevó la imposibilidad de captar las economías de escala propias de este tipo de industria y, en consecuencia, sería el responsable de la poca difusión de la electricidad en España.

Para probar lo antedicho, Antolín utiliza los datos aportados por su propia investigación -Antolín (1989) - sobre la empresa Hidroeléctrica Ibérica, pionera de la electrificación española y cuyo primer objetivo fue el suministro

7 El hecho de que la electricidad de origen hidráulico resultara en España más barata que el carbón, en términos relativos, no significa que el precio del fluido eléctrico fuera inferior al vigente en otros países. Lo era, probablemente, respecto a aquellos en los que predominaba la electricidad térmica, pero no respecto a otros que disponían de mejores condiciones hidráulicas. Desafortunadamente, la existencia de una multiplicidad de precios distintos según el tipo de consumo impide una comparación plenamente fiable.

${ }^{8}$ El cálculo correcto debería relacionar el producto industrial con el consumo de electricidad por la industria, pero la falta de información para todos los casos lo impide. El indicador que presentamos sería insesgado si la proporción del consumo industrial de electricidad respecto al consumo total fuera la misma en todos los países. 
de electricidad de origen hidráulico al País Vasco. Hacia 1930, esta compañía detentaba el 50 por 100 de la potencia total instalada para la producción de electricidad con destino al consumo en aquella zona, estando el resto en manos de diversas empresas menores, lo que le parece a Antolín prueba suficiente de los débiles cambios experimentados por el sector eléctrico español durante las dos décadas precedentes.

En este caso, las comparaciones internacionales resultan mucho más complejas, y la propia Francesca Antolín no presenta evidencia al respecto. Algunos indicios nos permiten pensar, sin embargo, que su hipótesis resulta, cuanto menos, arriesgada. La mejora de productividad que la electrificación puede aportar al conjunto de la economía vendrá, en buena parte, dada por su capacidad de ofrecer energía a un precio inferior al hasta ese momento vigente. Como hemos visto más arriba, parece claro que en España la ventaja relativa de la electricidad era muy superior a la que se daba en Estados Unidos y otros países. Nótese que esto tiene menos que ver con la dimensión de las plantas productoras que con el tipo de energía primaria que puede utilizarse. El factor dimensión sólo es relevante si comparamos instalaciones del mismo tipo. Hacia 1930, el 90 por 100 de la electricidad comercial producida en España provenía de plantas hidráulicas. En Estados Unidos, en esos mismos años, la proporción alcanzaba tan sólo el 30 por $100^{9}$.

Respecto a la transformación experimentada por el sector eléctrico español, la afirmación pesimista de Antolín carece, en mi opinión, de fundamento. Para dar tan sólo un ejemplo, en 1910, según los datos oficiales, las centrales de más de $1.000 \mathrm{kw}$ de potencia no pasaban de la treintena, eran en su mayoría térmicas y representaban en torno a un tercio de la potencia total registrada. En 1930 . las tres compañías más importantes —el grupo Barcelona Traction, Hidroeléctrica Española e Hidroeléctrica Ibérica - controlaban directamente en torno al 45 por 100 de la potencia instalada. Si tenemos en cuenta las vinculaciones que tenían las dos últimas empresas citadas entre sí y con otras a través del Banco de Vizcaya, podemos afirmar que este grupo y el controlado por la Barcelona Traction representaban conjuntamente más de la mitad del sector eléctrico español. En ese mismo año, 1930, se hallaban en funcionamiento 34 centrales de más de $10.000 \mathrm{CV}$ de potencia -24 de ellas hidroeléctricas- que representaban más del 60 por 100 de la potencia total ${ }^{10}$. Datos de carácter financiero o de cualquier otro orden vendrían igual. mente a cónfirmar la opinión unánime de los contemporáneos que veían en

- Cifras del Anuario Estadistico de España y del Bureau of Census (1976), p. 506, series $15-18$.

10 Datos de Ministerio de Fomento, Estadística de la industria eléctrica española a fin de 1910, y de Sintes Olives y Vidal Burdils (1933). 
el desarrollo del sector eléctrico uno de los fenómenos más llamativos de la España de entreguerras.

\section{Los inicios de la electricidad en España: una recapitulación}

Se han expuesto hasta aquí las principales discrepancias que, en mi opinión, explican la existencia de dos visiones contrapuestas de las primeras fases de la electrificación en España. Creo haber aportado elementos suficientes para poder reafirmar la hipótesis planteada en trabajos anteriores. Cuando, en los primeros años del presente siglo, nuevos hallazgos técnicos permitieron el transporte a largas distancias del fluido eléctrico, se abrió para España la posibilidad de mejorar notablemente su estructura energética. La nueva tecnología permitió el uso intensivo de un recurso relativamente abundante en España: la energía hidráulica. Dado el elevado coste que tenía aquí el carbón mineral, base hasta entonces del suministro energético, los precios de la electricidad producida por medios hidráulicos fueron desde el primer momento muy ventajosos en la mayoría de los usos industriales. Como consecuencia de ello, la electrificación española fue, en términos relativos, más intensa que la de otros países más avanzados, incluidos los Estados Unidos. El sector eléctrico experimentó a lo largo de este proceso una profunda transformación. De una estructura extremadamente atomizada, tanto desde el punto de vista de las dimensiones de las empresas como de las instalaciones de producción, se pasó a una estructura muy concentrada, en la que al final dos grupos controlaban más de la mitad de toda la potencia instalada.

En definitiva, creo que el proceso de electrificación que se produjo durante el primer tercio del siglo $\mathrm{xx}$ es uno de los pocos ejemplos de aprovechamiento rápido y profundo de una innovación tecnológica que podemos hallar en la historia económica de la España contemporánea.

\section{REFERENCIAS}

ANTolín, F. (1988): «Electricidad y crecimiento económico. Los inicios de la electricidad en España», Revista de Historia ECONÓmiCA, año VI, núm. 3, pp. 635-655.

- (1989): «Hidroeléctrica Ibérica y la electrificación del País Vasco», Revista de Economia Pública, núm. 5. (4), pp. 107-130.

Bureau of Census (1933): Abstract of the Fifteenth Census of the United States, Washington.

- (1976): Historical Statistics of the United States, Washington. 
Coll, S. (1987): «Primera parte, 1770-1936», en S. Coll y C. Sudrià, El carbón en España, 1770-1961. Una bistoria económica, Madrid.

Darmstadter, J., et al. (1971): Energy in the World Economy. A statistical review of trends in output, trade and consumption since 1925, Baltimore.

Devine, W. D. (1983): «From Shafts to Wires: Historical Perspective on Electrification», Journal of Economic History, vol. 43, núm. 2, pp. 344-372.

Maddison, A. (1982): Phases of Capitalist Development, Oxford.

Maluquer DE Motes, J. (1983): «L'electricitat», en J. Nadal, J. Maluquer de Motes, A. Carreras y C. Sudrià, Producció $i$ consum d'energia en el creixement econòmic modern: el cas català (inédito).

- (1985): «Cataluña y el País Vasco en la industria eléctrica española, 1901-1935», en M. González Portilla, B. Riquer y J. Maluquer de Motes (eds.), Industrialización y nacionalismo. Análisis comparativos, Bellaterra (Barcelona), pp. 239-252.

- (1987): «L'électricité, facteur de développement économique en Espagne: 1900-1936», en Un siècle d'électricité dans le monde, 1880-1980, París, pp. 57-67.

Mitchell, B. R. (1975): European Historical Statistics, 1750-1970, Londres.

Prados, L. (1987): De imperio a nación. Crecimiento y atraso económico en España (1780-1930), Madrid.

Schurr, S., y NetscherT, B. (1960): Energy in the American Economy 1850-1975, Baltimore.

Sintes Olives, F. F., y Vidal Burdils, F. (1933): La industria eléctrica en España, Barcelona.

Sudrià, C. (1987 a): "Un factor determinante: la energía», en J. Nadal, A. Carreras y C. Sudrià (eds.), La economía española en el siglo XX. Una perspectiva bistórica, Barcelona, pp. 313-363.

- $(1987 b)$ : «Les restrictions a la consommation d'électricité en Espagne pendant l'aprèsguerre: 1944-1954", en Un siècle d'électricité dans le monde, 1880-1980, París, pp. 425435.

- (1987c): «El dilema energètic en el creixement econòmic català», Revista Económica de Catalunya, núm. 4, pp. 88-100.

- (1989): «L'energia: de l'alliberament hidroelèctric a la dependència petroliera», en Història Econòmica de la Catalunya Contemporània. V. s. XX. Població, agricultura $i$ energia, Barcelona.

Woolf, A. G. (1984): «Electricity, Productivity and Labor Saving: American Manufacturing, 1900-1929", Explorations in Economic History, núm. 21, pp. 176-191. 